\title{
Atomic scale microstructures of high-k HfSiO thin films fabricated by magnetron sputtering
}

\author{
Etienne Talbot ${ }^{\mathrm{a}, *}$, Manuel Roussel $^{\mathrm{a}}$, Larysa Khomenkova ${ }^{\mathrm{b}}$, Fabrice Gourbilleau ${ }^{\mathrm{b}}$, Philippe Pareige ${ }^{\mathrm{a}}$ \\ a Groupe de Physique des Matériaux (GPM), Université et INSA de Rouen, UMR CNRS 6634, Av. de l'Université, BP 12, 76801 Saint Etienne du Rouvray, France \\ ${ }^{\mathrm{b}}$ Centre de Recherche sur les Ions, les Matériaux et la Photonique (CIMAP), CEA/CNRS/ENSICAEN/UCBN, 6 Bd. Maréchal Juin, 14050 Caen Cedex 4, France
}

\begin{abstract}
A B S T R A C T
High-k hafnium-silicate films were deposited by RF magnetron sputtering approach on silicon wafer. The microstructure has been investigated using the combination of transmission electron microscopy and atom probe tomography. It was evidenced that the elaborated $\mathrm{HfSiO}$ thin films subsequently annealed at $950^{\circ} \mathrm{C}$ during 15 min leads to a complex phase separated nanostructure where silica, hafnia and silicon nanoclusters coexist. The formation of silicon nanoclusters in hafnia-based host was never reported before. The results demonstrate the capability of RF magnetron sputtering to pave the way for realization of nanomemory devices based on silicon clusters embedded in high-k matrix.
\end{abstract}

\section{Introduction}

The development and improvement of nanoclusters based devices compatible with the silicon-based CMOS technology is a large and active research area [1]. One of the specific topics deals with group IV semiconductor nanoclusters diluted in dielectric matrix for photonic and microelectronic devices, for example, for third generation of photovoltaic cells or nanomemory devices. However, in the last case, silicon dioxide $\left(\mathrm{SiO}_{2}\right)$ met its physical limit as a gate dielectric (calculated to be $0.7 \mathrm{~nm} \mathrm{[2])} \mathrm{due} \mathrm{to} \mathrm{an}$ unacceptable leakage current for reliable and high performance devices. To overcome this problem, new materials with a higher dielectric constant (high-k materials) have been proposed. Among them, hafnia $\left(\mathrm{HfO}_{2}\right)$ and its silicates appear as the most promising high-k dielectrics to replace silica. They offer good chemical and thermal stability, as well as the good interfacial quality with $\mathrm{Si}$ channel [2-5]. Nevertheless, from the point of view of the electrical properties of the devices, a deeper analysis of the high- $\mathrm{k}$ dielectrics is required. In this regard, to reveal the nature of the high-k layer/silicon channel interface is one of the main tasks to achieve the high reliability of the devices.

An application of high-k materials was also extended to floating gate non-volatile memory devices containing semiconductor nanoclusters embedded in $\mathrm{SiO}_{2}$ host [6]. However, till present time the formation of such clusters in high-k host is questionable. In spite of reported elaboration and fine characterization of Si-rich$\mathrm{HfO}_{2}$ systems over a large composition range, any formation of Si nanoclusters was not revealed [7-9]. In the present article, the atomic scale characterization of HfSiO thin films prepared by magnetron sputtering has been performed by transmission electron microscopy and atom probe tomography. We demonstrate that the growth of nanometric silicon clusters can occur in hafnium silicate elaborated by magnetron sputtering.

\section{Experimental technique}

\subsection{Magnetron sputtering elaboration}

A $230 \mathrm{~nm}$ high-k HfSiO thin films were deposited on a (100) ptype CZ Si wafers by radio-frequency (RF) magnetron co-sputtering of $\mathrm{HfO}_{2}(99.99 \%)$ target topped with Si chips placed on the electron 'race track'. The films were fabricated under a RF power density of $0.74 \mathrm{~W} / \mathrm{cm}^{2}$ and a substrate temperature of $100^{\circ} \mathrm{C}$ in mixed $\mathrm{Ar}-\mathrm{H}_{2}$ plasma. The use of hydrogen is due to its ability to remove the oxygen from plasma and consequently, to reach higher Si excess in the layers. More details on the reactive fabrication process can be found elsewhere $[10,11]$. The total plasma pressure was kept at 0.04 mbar. Finally, the sample was annealed under a nitrogen flow in a conventional furnace at $950{ }^{\circ} \mathrm{C}$ during $15 \mathrm{~min}$. 


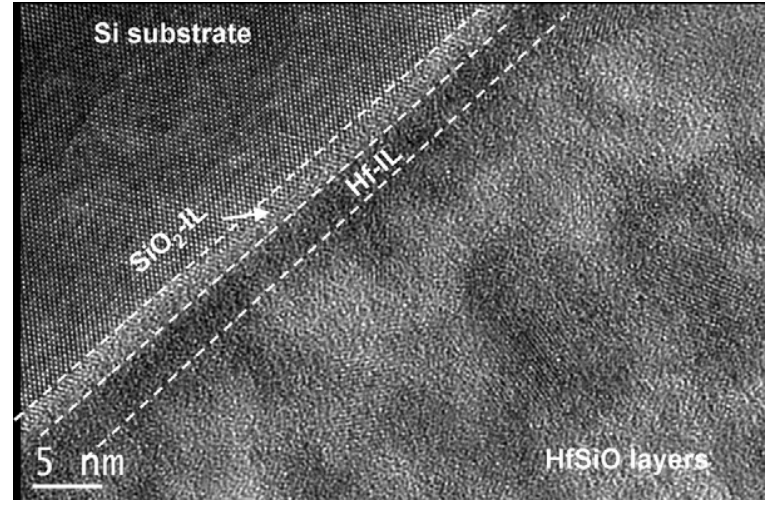

Fig. 1. Cross-section HR-TEM micrograph of the HfSiO layer. $\mathrm{SiO}_{2}$-IL and Hf-IL denote $\mathrm{SiO}_{2}$ rich and $\mathrm{Hf}$ rich interface layers respectively.

\subsection{Atom probe tomography}

Atom probe tomography (APT) is a three dimensional highresolution analytic microscopy offered spatial mapping of atoms in materials [12]. Nowadays, this technique is increasingly used for the study of semiconductor and dielectric materials $[13,14]$. The APT technique is based on the field evaporation of surface atoms from tip-shaped specimen with a curvature radius lower than $50 \mathrm{~nm}$. Tips preparation was realized in a dual-beam FIB-SEM, using a lift out method following by annular milling procedure [15]. The experiments were performed with in a Laser Assisted Wide Angle Atom Probe (LAWATAP - CAMECA) at a temperature of $80 \mathrm{~K}$, using a laser-wavelength of $\lambda=343 \mathrm{~nm}$ with femtosecond pulses duration $(350 \mathrm{fs}, 50 \mathrm{~nJ})$ at $100 \mathrm{kHz}$ pulse rate.

\subsection{Transmission electron microscopy}

High-resolution Transmission Electron Microscopy (HR-TEM) was performed in a JEOL ARM-200F operated at $200 \mathrm{kV}$. TEM lamellas were prepared by conventional in situ lift-out technique.

\section{Results and discussion}

\subsection{TEM experiments}

Fig. 1 shows a cross-sectional HR-TEM image of the HfSiO films. The revealed bright and dark contrast can be associated with Si-rich (bright regions) and Hf-rich (dark ones). This gives a direct evidence of Hf segregation process already reported in the literature [5,7]. The Hf-rich regions appear to be crystallized. In addition, the interfacial region between $\mathrm{HfSiO}$ film and $\mathrm{Si}$ substrate demonstrates the presence of two layers, i.e. 2-nm thick $\mathrm{SiO}_{x}$ layer followed by 3-nmthick Hf-rich layer parallel to the Si substrate. Similar structure has been already observed in the case of ultrathin HfSiO layers [5].

\subsection{APT experiments}

Atom probe tomography is one of the most accurate techniques to measure chemical compositions in nanostructured materials. The mass spectrum obtained on the HfSiO sample is shown in Fig. 2. All the mass peaks are identified and well associated to the chemical species. In regard with the dielectric nature of the sample, we should note that the mass resolution is relatively $\operatorname{good}\left(m / \Delta m=400\right.$ at full width half maximum on the $\mathrm{O}_{2}{ }^{2+}$ peak). Moreover, hafnium atoms were detected as molecular ions $\left(\mathrm{HfO}_{2}{ }^{3+}\right.$ and $\mathrm{HfO}_{2}{ }^{2+}$ ). Finally, no $\mathrm{Ga}$ atoms have been detected, indicating that the FIB-SEM use for tip preparation has not created any damage in the microstructure. The chemical composition has

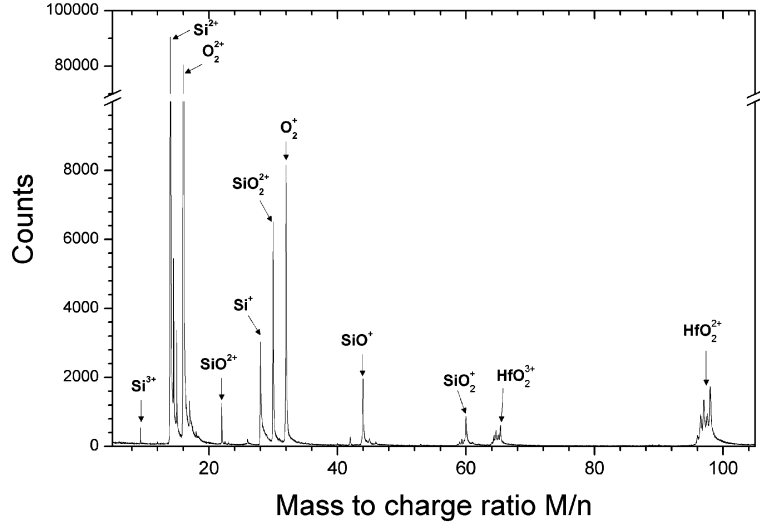

Fig. 2. Mass spectrum collected during APT experiment on the HfSiO layers.

been determined from the mass spectrum and the $\mathrm{Si}, \mathrm{O}$ and $\mathrm{Hf}$ contents are respectively estimated to $X_{\mathrm{Si}}=30.6 \pm 0.3$ at.\%, $X_{\mathrm{O}}=61.5 \pm 0.3$ at.\% and $X_{\mathrm{Hf}}=7.9 \pm 0.3$ at.\%.

Fig. 3 shows the 3D reconstructed spatial distribution of $\mathrm{Si}, \mathrm{O}$ and $\mathrm{Hf}$ atoms in the HfSiO thin layers. Silicon and Hafnium atoms are non-homogenously distributed in the analysed volume indicating clearly phase separation upon sample annealing. The presence of Hf-rich and Si-rich zones is revealed and is consistent with the microstructure deduced from TEM experiments (Fig. 1).

In order to identify the microstructure of the HfSiO layer and to measure the composition of the different chemical zones and their spatial distributions, we have calculated iso-concentration surface on $\mathrm{Hf}$ and Si atoms. Fig. 4a shows the surface for $X_{\mathrm{Hf}}>25$ at.\% (in the blue) and $X_{\mathrm{Si}}>33$ at.\% (in the red). These data provide the evidence of phase separation between $\mathrm{SiO}_{2}$-rich and $\mathrm{HfO}_{2}$-rich phases induced by the annealing treatment. Moreover, increasing the concentration threshold of iso-concentration (Fig. $4 \mathrm{~b}$ where the threshold are $X_{\mathrm{Hf}}>40$ at.\% and $X_{\mathrm{Si}}>75$ at.\%) does not evidence $\mathrm{Hf}$ zones containing more than 40 at.\% but only very rich-Si clusters. Fig. $4 \mathrm{c}$ displays a top view in a selected region of the analysed volume which contains the different chemical zones mentioned previously. Compositions of the three chemical regions were measured and reported in Table 1. We should note that APT experiment can lead to a bias in the concentration measurement in the case of multi-phase sample analysis. This effect is due to the difference in evaporation field between the different phases [16]. All the concentration
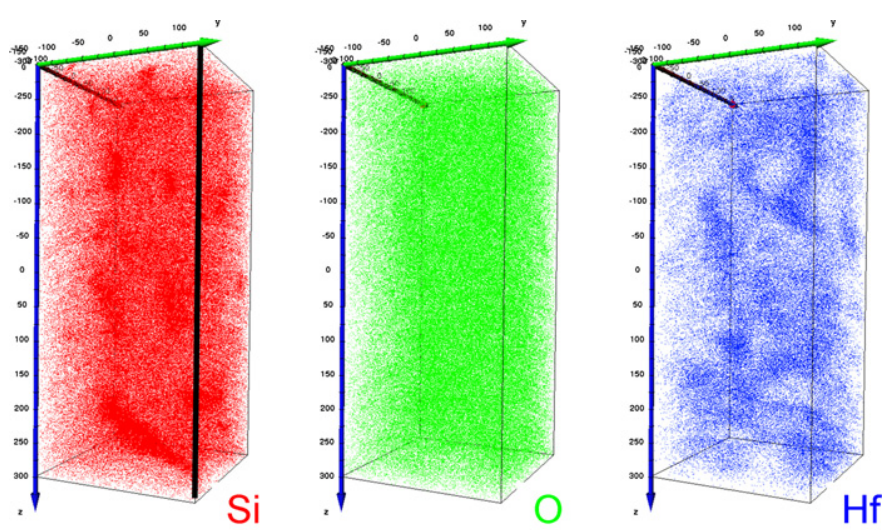

\section{$10 \mathrm{~nm}$}

Fig. 3. 3D chemical maps ( $\mathrm{Si}$ in red, $\mathrm{O}$ in green and $\mathrm{Hf}$ in blue) of the HfSiO laye obtained by APT analyses. For clarity of the figures, only $50 \%$ of Si and O are represented. The volume is $25 \mathrm{~nm} \times 25 \mathrm{~nm} \times 60 \mathrm{~nm}$. (For interpretation of the references to color in this figure legend, the reader is referred to the web version of the article.) 

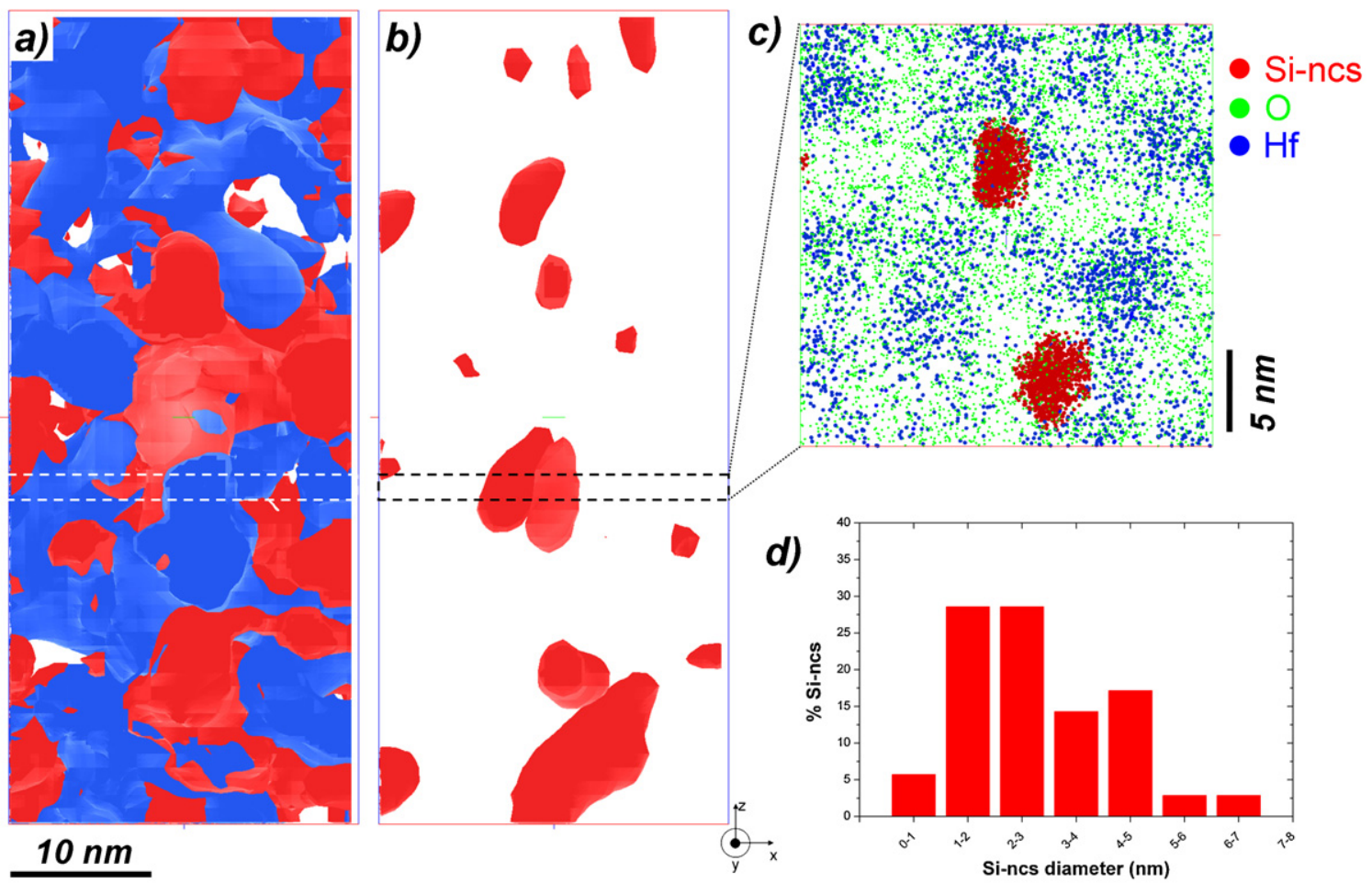

Fig. 4. 3D chemical isoconcentration surfaces of hafnium and silicon extracted from the 3D chemical maps shown in Fig. 3. The thresholds on Hafnium and Silicon concentration are respectively (a) $X_{\mathrm{Hf}}>25$ at.\% and $X_{\mathrm{Si}}>33$ at.\%, (b) $X_{\mathrm{Hf}}>40$ at.\% and $X_{\mathrm{Si}}>75$ at.\%. (c) 3D atomic mapping in selected region (dash rectangle - volume $25 \mathrm{~nm} \times 25 \mathrm{~nm} \times 1.5 \mathrm{~nm}$ ). For clarity, only silicon atoms belonging to pure Si-ncs are shown. (d) Size distribution of the Si-ncs diameter in the HfSiO layers. (For interpretation of the references to color in this figure legend, the reader is referred to the web version of the article.)

Table 1

Chemical composition in different chemical zones.

\begin{tabular}{lcccl}
\hline Zone & $X_{\mathrm{Si}}($ at.\%) & $X_{\mathrm{O}}($ at.\%) & $X_{\mathrm{Hf}}$ (at.\%) & Phase \\
\hline Hf-rich & 0.0 & $68.2 \pm 0.2$ & $31.8 \pm 0.2$ & $\mathrm{HfO}_{2}$ \\
Si-rich & $32.5 \pm 0.2$ & $66.2 \pm 0.2$ & $1.3 \pm 0.2$ & $\mathrm{SiO}_{2}$ \\
Si-ncs & $98.8 \pm 0.2$ & $1.2 \pm 0.2$ & 0.0 & Si-ncs \\
\hline
\end{tabular}

presented in this study has been corrected by applying the procedure developed on similar compounds by Talbot et al. [13].

The presence of a dual matrix composed of $\mathrm{SiO}_{2}$ and $\mathrm{HfO}_{2}$ is clearly demonstrated confirming the phase separation predicted by the pseudo binary phase diagram $\mathrm{HfO}_{2}-\mathrm{SiO}_{2}$ which shows a miscibility gap. In the same way, precipitation of pure Si nanoclusters homogenously distributed in the volume with a density of $2.9 \pm 0.4 \times 10^{17} \mathrm{Si}$-ncs $/ \mathrm{cm}^{3}$ is also demonstrated. The Si nanoclusters density was determined by counting the number of clusters detected and divided it by the volume of matter analysed. Accurate size distribution of the Si nanoclusters extracted from APT analysis is presented in Fig. $4 \mathrm{~d}$. The size ranges from 0.9 to $6.5 \mathrm{~nm}$ with a mean diameter of $2.8 \mathrm{~nm}$.

Phase separation of hafnium silicate has been intensively studied and only $\mathrm{SiO}_{2}$ and $\mathrm{HfO}_{2}$ formation were reported. Nevertheless, such investigations have been performed on high content of hafnium in Hf silicate. The APT study of low content with direct evidence of Si-ncs indicates unambiguously that atomic structure of the film after thermal treatment is strongly dependant of the composition of the film.

\section{Conclusion}

In summary, the microstructure of high-k Hf-silicate thin layers grown using magnetron sputtering was investigated by TEM and APT. Phase separation between silica and hafnia after $950{ }^{\circ} \mathrm{C}$ annealing was clearly evidence and leads to the growth of pure Si nanoclusters in the layer. Such Si-ncs, which have not been observed by TEM, are only revealed by APT analysis demonstrating the benefit of dual APT-TEM investigations for nanoclusters based $\mathrm{Hf}$ silicate materials. Moreover, these results validate reactive magnetron sputtering approach for high-k materials elaboration and open the way to produce new nanomemory devices.

\section{Acknowledgments}

This work was supported by the Upper Normandy Region and the French Ministry of Research in the framework of Research Networks of Upper-Normandy. The growth of the layer is supported by the French National Research Agency (ANR) through Nanoscience and Nanotechnology Program (NOMAD Project no. ANR-07-NANO022-02) and, for one of the authors (L. Khomenkova) by the Conseil Régional de Basse Normandie through the CPER project Nanoscience axe (2007-2013).

\section{References}

[1] S. Tiwari, F. Rana, H. Hanafi, A. Harstein, E.F. Crabbé, K. Chan, Appl. Phys. Lett. 68 (1996) 1377.

[2] G.D. Wilk, R.M. Wallace, J.M. Anthony, J. Appl. Phys. 89 (2001) 5243.

[3] G.D. Wilk, R.M. Wallace, Appl. Phys. Lett. 74 (1999) 2854

[4] M.A. Quevedo-Lopez, M. El-Bouanani, B.E. Gnade, R.M. Wallace, M.R. Visokay, M. Douglas, M.J. Bevan, L. Colombo, J. Appl. Phys. 92 (2002) 3540.

[5] L. Khomenkova, C. Dufour, P. Coulon, C. Bonafos, F. Gourbilleau, Nanotechnology 21 (2010) 095704.

[6] W. Li, R. Jia, C. Chen, M. Liu, H.F. Li, C.X. Zhu, S. Long, ECS Trans. 18 (2009) 1071.

[7] S. Stemmer, Y. Li, B. Foran, P.S. Lysaght, S.K. Streiffer, P. Fuoss, S. Seifert, Appl. Phys. Lett. 83 (2003) 3141

[8] Y.Q. Wang, J.H. Che, W.J. Yoo, Y.C. Yeo, J. Appl. Phys. 98 (2005) 013536.

[9] J. Liu, X. Wu, W.N. Lennard, D. Landheer, Phys. Rev. B 80 (2009) 041403. 
[10] C. Ternon, F Gourbilleau, X Portier, P. Voivenel, C Dufour, Thin Solid Films 419 (2002) 5 .

[11] L. Khomenkova, X. Portier, J. Cardin, F. Gourbilleau, Nanotechnology 21 (2010) 285707.

[12] D. Blavette, B. Deconihout, A. Bostel, J.M. Sarrau, M. Bouet, A. Menand, Rev. Sci. Instrum. 64 (1993) 2911.
[13] E. Talbot, R. Lardé, F. Gourbilleau, C. Dufour, P. Pareige, Europhys. Lett. 87 (2009) 26004

[14] R. Lardé, E. Talbot, P. Pareige, H. Bieber, G. Schmerber, S. Colis, V. PierronBohnes, A. Diana, J. Am. Chem. Soc. 133 (2011) 1451.

[15] G.B. Thompson, M.K. Miller, H.L. Fraser, Ultramicroscopy 100 (2004) 25

[16] D. Blavette, F. Vurpillot, P. Pareige, A. Menand, Ultramicroscopy 89 (2001) 145. 